\title{
Clinical potential of DAC-70 sol
}

\author{
Akio Sugitachi ${ }^{* 1}$, Naoko Takahashi ${ }^{1}$ and Yoshimori Takamori ${ }^{2}$ \\ ${ }^{1}$ Department of Surgery, Iwate Medical University, Morioka 020-8505, Japan \\ ${ }^{2}$ Koyo Chemical Co. Ltd, Osaka 530-0051, Japan
}

\begin{abstract} 70) sol to provide a clinically useful biodegradable material.

\section{Introduction}

We applied the DAC-70 sol as a drug vehicle in drug delivery system (DDS). To cyto-histologically identify cancer cells, we used the agent in a photodynamic diagnosis (PDD) together with aminolevulinic acid (ALA). We devised a novel endoscopic marker using both DAC70 sol and carbon powder (CP). The DAC-70 sol was also modified to an anticancer agent and loco-regional enema. In this paper, we summarized potential of such DAC-70 sol in clinical applications.
\end{abstract}

Chitins and chitosans are biodegradable polysaccharides mainly purified from crab crusts. The authors newly prepared seventy percent deacetylated chitosan (DAC-

\section{Materials and methods}

Seventy percent specially prepared deacetylated chitosan powder (DAC-70: Koyo Chemical Co. Ltd. Osaka, Japan) [1], Glycerol Phosphate Disodium Salt n-Hydrate (GP), Amino levulinic acid (ALA) and Acetylsalicylic Acid (ASA: Wako Pure Chemical Co. Ltd. Osaka, Japan), HCT 116 cells and HeLa cells (RIKEN BRC Cell Bank, Tsukuba, Japan), RPMI 1640 cell culture medium (RPMI) and Fetal bovine serum (FBS: Gibco, Life Technologies Corp, Grand Island, NY, USA), Carbon powder (CP: Unitika Co. Ltd. Osaka, Japan), blue light source (SOLARFORCE, L2P: Hong Kong). Anticancer drugs: cis-platinum (CDDP; Nippon KAYAKU Co. Ltd, Tokyo, Japan), fluorouracil (5FU; Kyowa Hakko Kogyo Co. Ltd, Tokyo, Japan) and gemcitabine (GEM; Lilly Japan Co. Ltd, Kobe, Japan). Other reagents used were analytical grade.

(1) First, we prepared DAC-70 sol. The preparation procedures have already been published [2], (Figure 1).

(2) The DAC-70 sol was applied as a biodegradable drug vehicle in drug delivery system (DDS); namely, the novel sol was individually mixed with CDDP, 5FU, GEM, ASA and ALA to form drug conjugates. Concentration of each drug released from the mixture into saline solution at $37^{\circ} \mathrm{C}$ was periodically measured to examine in vitro release profile.

(3) To investigate anticancer effects of the DAC-70 sol, we cultured two different types of cancer cell line in the RPMI medium with FBS containing the DAC-70 sol and then, examined in vitro anti-proliferative activity of each cell with WST assay. In in vivo anti-cancer studies, we directly administered the DAC-70 sol into malignant tumors or ascites of cancer-bearing nude mice to record changes of tumor size or survival time of the treated animals.
(4) In an attempt to examine in vivo healing effects for inflammatory bowel diseases (IBD), we induced colon ulcers in SD rats by intracolonic injection of $10 \%$ acetic acid solution. Then, ASA/DAC-70 sol was topically given by enema on the injured areas. The therapeutic effects with the sol-enema-treatment were macroscopically and microscopically evaluated.

In all animal experiments, we faithfully abided by the ethical rules in our institute.

(5) We applied the DAC-70 sol in PDD; namely, ALA was mixed with DAC-70 sol to prepare a novel photosensitizer (ALA/DAC$70 \mathrm{sol}$ ). A testing sample was soaked in the sol for a while prior to microscopically identify reddish-pink fluorescence specifically emitted in cancer cells under visible blue light. Our newly devised endoscopic marker was prepared and its efficacy was examined as follows; viz. the carbon powder (CP) was mixed with DAC-70 sol (CP/DAC-70 sol), which was injected into submucosal layer of experimental SD rat gastric wall to confirm whether the novel sol was clinically applicable or not. The injected sites and the around organs were macroscopically and histo-pathologically examined to evaluate in vivo functions and side effects with the novel marker. Using human colons resected for surgical therapy, we also carried out an ex vivo study to examine clinical utility of the sol as an injectable marker.

\section{Results}

(1) DAC-70 sol was viscoelastic and injectable, in which various drugs could be easily dissolved to form homogeneous mixed solutions. While the CPs were indissoluble into the sol, but could be suspended by stirring to become homogeneous $\mathrm{CP} / \mathrm{DAC}-70$ sol, which was also injectable via a $21 \mathrm{G}$ needle.

(2) Release profile of each drug from the conjugated DAC-70 sol showed a sustained release pattern. About $80 \%$ of GEM and ALA were gradually released from the drug carriers in initial 12 hours and such

Correspondence to: Akio Sugitachi, MD. Dept. of Surgery, Iwate Medical University, Morioka 020-8505, Japan, Fax: +81-19-651-7166; E-mail: akiosugi@iwate-med.ac.jp

Received: April 25, 2016; Accepted: May 09, 2016; Published: May 12, 2016 
levels were maintained for following 24 hours. More than $90 \%$ of ASA and CDDP were delivered from the conjugated sols within 6 hours and the levels were also maintained for more than 24 hours. The 5-FU showed an initial bursting. Almost all amount of 5-FU loaded in the DAC-70 sol was delivered within 3 hours. Each release profile of the drug loaded in the DAC-70 drug carrier was shown in Figure 2.

(3) In vitro proliferation of the HCT 116 and HeLa cells were completely suppressed in the culture medium containing DAC-70 sol. While, in vivo tumor regression by intra-tumor direct injection of DAC-70 sol revealed insufficient; namely, the HCT 116 tumors treated with the sol temporally reduced in volume, but finally regrowth of the tumors was encountered. Intra-abdominal administration of CDDP/ DAC-70 sols into malignant ascites-bearing mice suggested promising results [2], (Figure 3).

(4) Topical administration of the ASA/DAC-70 sol-enema showed favorable healing effects on the colonic ulcers induced with acetic acid. Survival time of the treated animals was significantly prolonged; namely, 7/11 rats treated with ASA/DAC-70 sol survived for longer than 4 weeks and 3/8 animals treated with DAC-70 sol also survived for

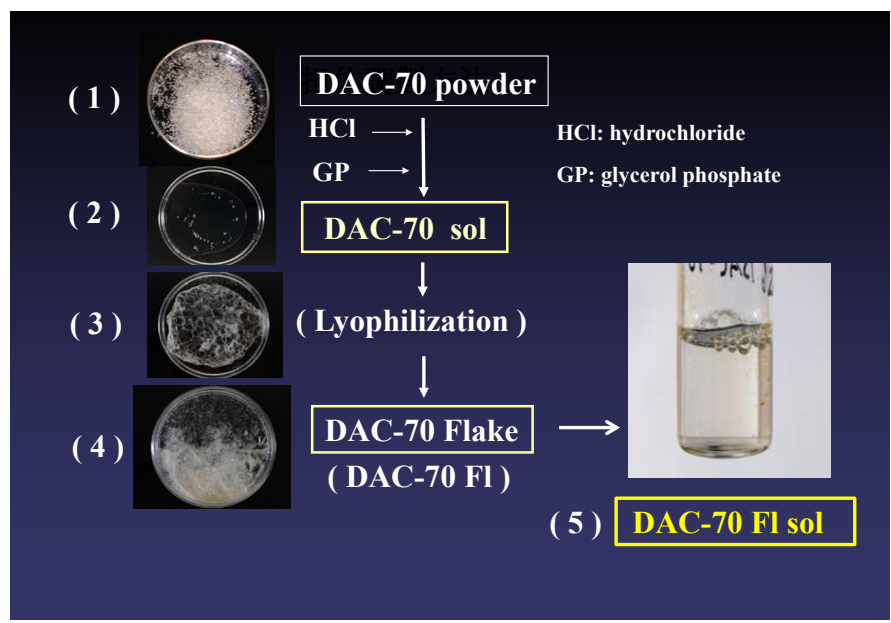

Figure 1. Preparation procedures of DAC-70 sol. The details have been reported in the reference 2 .

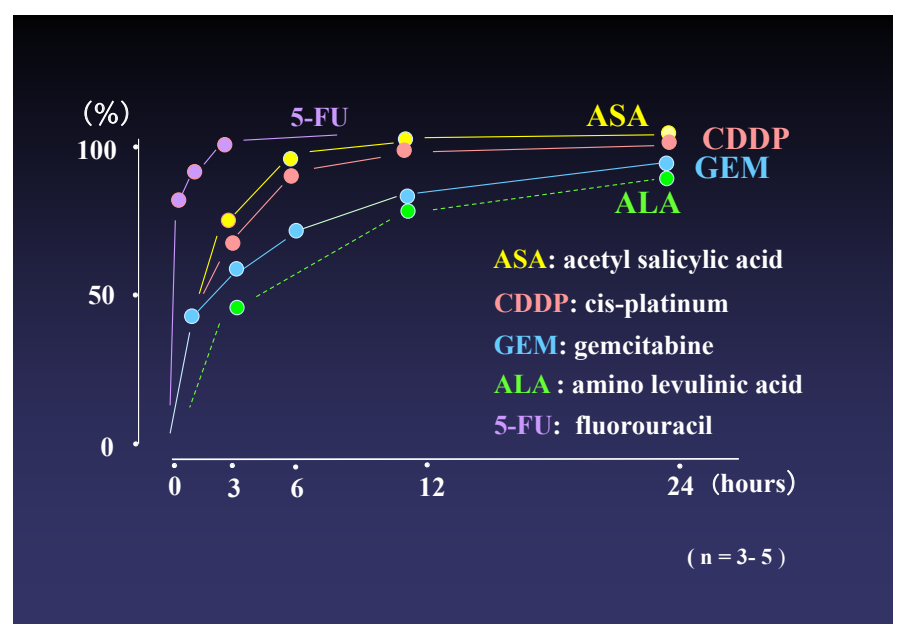

Figure 2. Release profile of each drug from the conjugate into physiological saline solution at $37^{\circ} \mathrm{C}$. ASA, CDDP, GEM and ALA showed sustained release suggesting clinical practice. 5-FU revealed initial bursting. Further ameliorative modification for slow-release will be required for clinical application of 5-FU.

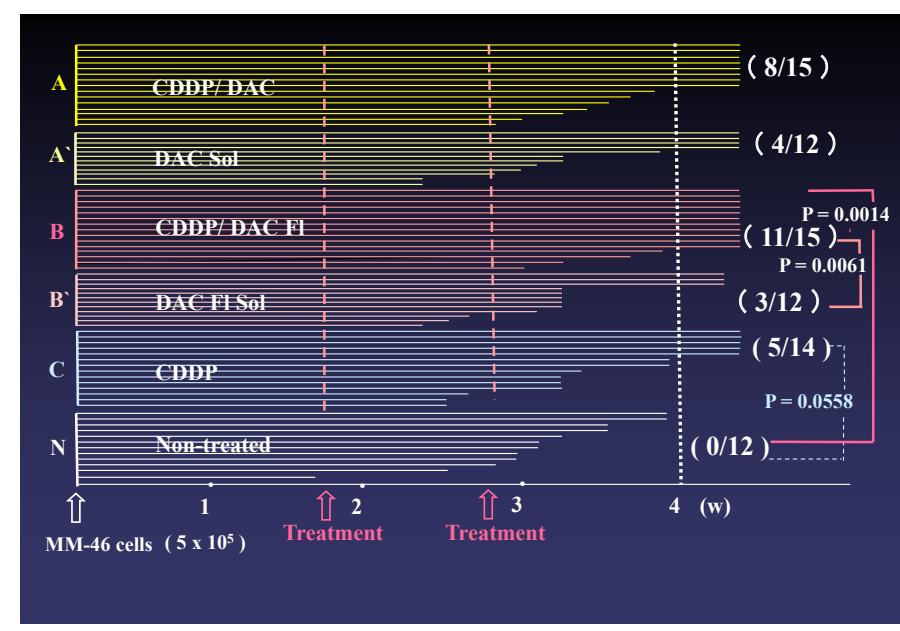

Figure 3. Survival time of malignant ascites-bearing animals

CDDP/DAC Fl-treated group (B) provided statistically superior efficacy to Nontreated group $(\mathrm{N})$. The (B) group also presented an advantageous $\mathrm{p}$ value to (B') group. Combination with the DAC-70 sol and CDDP would be useful in cancer chemotherapy.

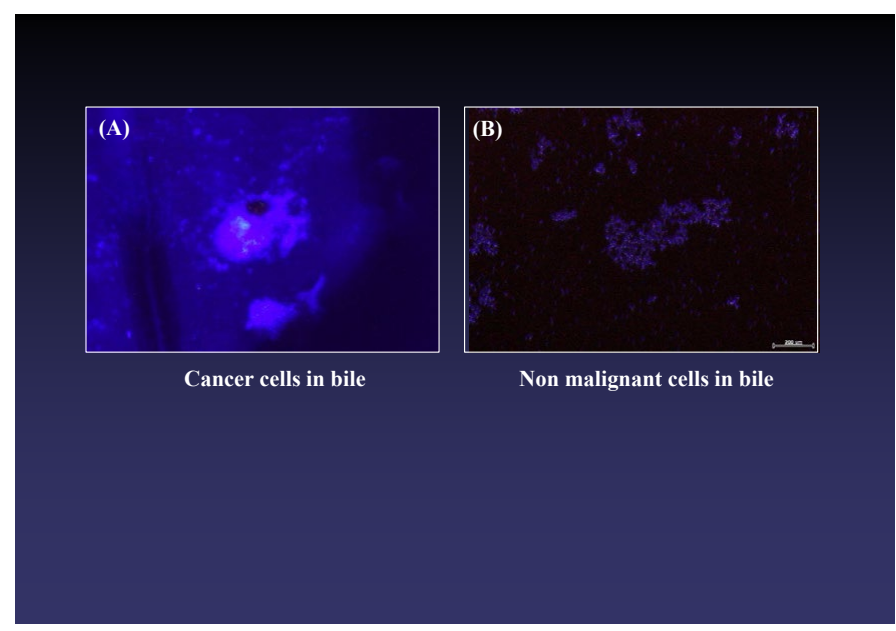

Figure 4. Microscopic findings of bile samples treated with ALA/DAC-70 sol. Cancer cells emitted specific reddish-pink fluorescence (A). No pink fluorescence was observed in the sample including no malignant cells (B).

longer than 4 weeks. No recurrence of ulcer formation was observed in these long-survived animals. All 10 animals received non treatment died of ulcer-perforation and/or massive colonic bleeding within 5 days.

(5) The ALA/DAC-70 sol well functioned as a microscopic indicator of cancer cells.

Using the novel photosensitizer, we could clinically find out a bile tract cancer which had been diagnostically intractable [3,4] (Figure 4). The CP/DAC-70 sols injected into human colons suggested practically useful marker showing pin-point markings [5], (Figure 5).

\section{Discussion}

Our newly prepared DAC-70 sol suggested for different kinds of clinical applications; e.g. it showed efficient results as drug carriers in our newly designed cancer chemotherapy and IBD treatment. Based on our in vivo studies, the sol would initially function as a transient loco-regionally adhesive antineoplastic agent or a promoter of wound healing by itself and then, each drug loaded in the sol would provide long-lasting original ability by its slow releasing. We considered this 
pharmacological system could reduce regular therapeutic dosage of each drug. In the cases of cancer chemotherapy, such would avoid side effects due to anticancer drugs. The sol also revealed clinical utility in diagnostic technique such as ALA-PDD or injectable pin-point marking.

We speculated that these hopeful results would attribute to some biologically favorable mechanisms endowed in the DAC-70 sol; namely, they were biodegradability, viscosity and fine structure of the sol. Since the DAC-70 sol is histo-compatible [6] and gradually degrades in situ, it will be safe for medical applications in human. In our in vivo studies, the experimental animals treated with DAC-70-related sols encountered no untoward events caused by the agents. Viscosity of the sol would play important roles in loco-regional accumulation and long-staying of the drugs loaded, by which we could obtain effective results both in our ASA/DAC-70 sol-treatment for IBD models and CP/DAC-70 sol markings. In our clinical case, we could find out a few cancer cells from bile sample mingled with many degenerated

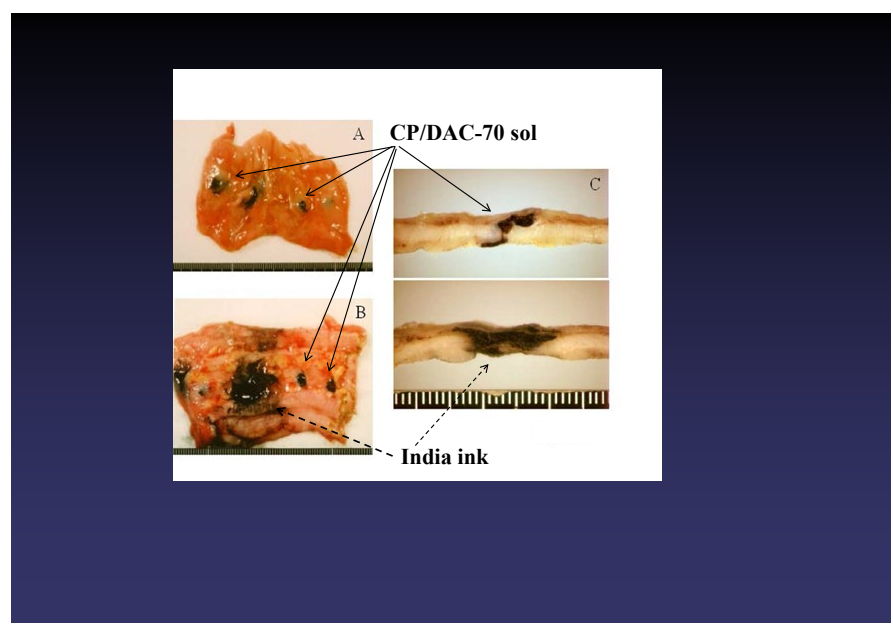

Figure 5. Macroscopic findings of human colons injected two different types of marker (CP/DAC-70 sol and India ink). CP/DAC-70 sol loco-regionally stayed at the site injected clearly indicating pin-point markings as shown in A, B and C. The India ink used as the control marker widely spread in the injected area, as shown in B and C.

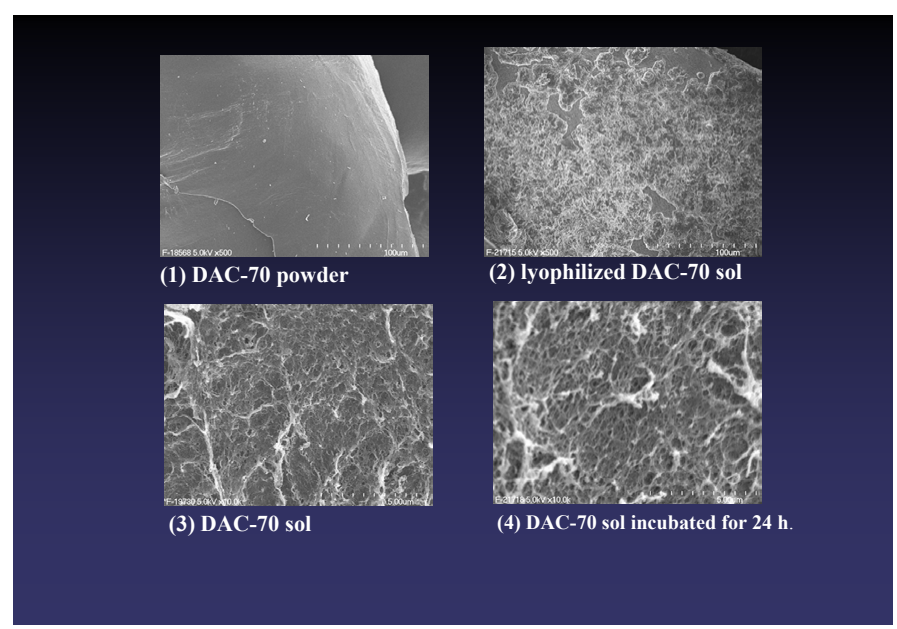

Figure 6. Scanning electron microscopic findings of DAC-70 materials in various steps of the preparation to DAC-70 sol. Surface of original DAC-70 powder was smooth (1). Such surface changed to elaborate mesh after lyophilization of the sol (2). DAC-70 sol showed fine network structure, which changed to rough hydrosol networks when incubated in liquid. cells and debris by cytological preparation procedures using the ALA/ DAC-70 sol. We considered the viscosity with the sol would serve as an adhesive and collecting agent for the cancer cells we wanted. Scanning electron microscopy revealed that our DAC-70 sol was constructed with assembly of fine networks (Figure 6). We assumed such networks would function as biodegradable reservoirs for various drug solutions, from which each drug loaded would be slowly delivered to provide a sustained release, and the drug carriers gradually degraded discharging their function to leave no remnants in the sites they had been placed.

Same kinds of biomedical studies on chitin and chitosan materials have been reported; e.g. wound healing dressings [7,8], hemostatic materials [9], drug carriers [10,11], etc. All these works are promising, though clinically used materials have been limited yet.

Safety in clinical applications of these kinds of materials should be most important.

For the preparation of clinically available biomedical chitins and chitosans, degree of deacetylation and endotoxin content in the materials are also seriously prescribed. As for deacetylation degree, $70 \%$ deacetylated chitosan (DAC-70) was reasonable for biomedical material [12]. The DAC-70 we used in these studies was specially prepared material with safety and high quality; namely, the concentration of endotoxin included in our DAC-70 was detected less than 1/10 amount regulated in our country [13]. Internationally, the American Society for Testing and Materials (ASTM) prescribes strict rules for safety [14]. We will confirm whether our DAC-70 materials conform to the ASTM rules or not.

We consider that our DAC-70 sol will be clinically potential in therapeutic DDS and microscopic and/or endoscopic diagnoses. The DAC-70 Flake (FAC-70 Fl) will especially suit for preparation of many different kinds of DAC-70-related sols. As the DAC-70 Fl forms fine dry granules, it can be easily transformed to injectable sol when immersed into liquid and such dry granular DAC-70 Fl is suitable for long preservation. All over clinical safety of our material is still pending.

\section{Acknowledgement}

The authors are grateful to Mr. Naoki Omura, Iwate Medical University, Morioka, Japan for technical assistance in the experiments.

\section{References}

1. Kurozumi S, Takamori Y, Fukuda M, Iwase H, Maeda M, et al. (2007) Studies on Development for Hemostatic Agent using Partially Deacetylated Chitin and Chitosan. Chitin Chitosan Res 13: 94-95.

2. Sugitachi A, Takahashi N, Kume K, Ohmori Y, Nishizuka S, et al. (2014) Topica administration of a newly devised anticancer agent: A novel concept of cancer chemotherapy. Integr Cancer Sci Thera 1: 57-60.

3. Ito N, Sugitachi A, Takahashi M, Makabe K, Kanno S, et al. (2013) Photodynamic diagnosis and preoperative chemotherapy for biliary tract cancer. Gan To Kagaku Ryoho 40: 1641-1643. [Crossref]

4. Sugitachi A, Otsuka K, Kimura K, Hakozaki M, Yaegasshi M, et al. (2015) Colorimaging histodiagnostic approach for cancer. Integr Mol Med 2: 231-233.

5. Sugitachi A, Otsuka K, Akiyama Y, Itabashi T, Asahi H, et al. (2006) A Novel Endoscopic Maker: Safety Experiments in the Rat Stomach. Endoscopy 38: 735-738. [Crossref]

6. Biomedical Materials from Chitin and Chitosan in Material Science of Chitin and Chitosan (eds. Uragami T and Tokura S), Kodansha, Japan and Springer, 2006, pp191218

7. Dai T, Tanaka M, Huang YY, Hamblin RM (2011) Chitosan preparations for wounds and burns: antimicrobial and wound healing effects. Expert Rev Anti Infect Ther 9 : 857-879. [Crossref] 
8. Baxter RM, Dai T, Kimball J, Wang E, Hamblin MR, et al. (2013) Chitosan dressing promotes healing in third degree burns in mice. Gene expression analysis shows biphasic effects for rapid tissue regeneration and decreased fibrotic signaling. J Biomed Mater Res A 101: 340-348. [Crossref]

9. Shikani AH, Chahine KA, Algudah MA (2011) Endoscopically guided chitosan nasal packing for intractable epistaxis. Am J Rhinol Allergy 25: 61-63. [Crossref]

10. Tan ML, Friedhuber AM, Dunstan DE, Choong PF, Dass RC (2010) The performance of doxorubicin encapsulated in chitosan-dextran sulphate microparticles in an osteosarcoma model. Biomaterials 31: 541-551. [Crossref]
11. Parveen S, Sahoo SK (2011) Long circulating chitosan/PEG blended PLGA nanoparticle for tumor drug delivery. Eur J Pharmacol 670: 372-383. [Crossref]

12. Nishimura K, Nishimura N, Nishi N, Saiki S, Tokura I, et al. (1984) Immunological activity of chitin and its derivatives. Vaccine 2: 93-99. [Crossref]

13. Kurozumi S, Kaga I, Takamori Y, Fukuda M, Furuike T, et al. (2011) Reduction of endotoxin in chitin and chitosan and method for measurement of endotoxin. Chitin Chitosan Res 17: 160-161.

14. Dornish M, Kaplan D, Skangrud O (2001) Standards and guidelines for biopolymers in tissue-engineered medical products: ASTM alginate and chitosan standard guides, American Society for Testing and Materials. Ann NY Acad Soci 944: 388-397. [Crossref]

Copyright: $\odot 2016$ Sugitachi A. This is an open-access article distributed under the terms of the Creative Commons Attribution License, which permits unrestricted use, distribution, and reproduction in any medium, provided the original author and source are credited. 